\title{
Bounded input bounded output stability for Lurie system with time-varying delay
}

\section{B. Wang ${ }^{1,2^{*}}$, J. Cheng ${ }^{3}$ and S.M. Zhong ${ }^{2}$}

"Correspondence:
coolbie@163.com
'Key Laboratory of Fluid and Power
Machinery of the Ministry of
Education, School of Electrical
Engineering and Electronic
Information, Xihua University,
Chengdu, P.R. China
2School of Applied Mathematics,
University Electronic Science and
Technology of China, Chengdu,
P.R. China
Full list of author information is
available at the end of the article

available at the end of the article

\begin{abstract}
This paper studies the bounded input bounded output stability for the Lurie system with time-varying delay. Utilizing the Lyapunov method and linear matrix inequality technology, new bounded input bounded output stability criteria are derived. The numerical simulation is carried out to show the system's dynamic response, and demonstrate the effectiveness of theoretical results.
\end{abstract}

Keywords: Time-varying delay; Stability; Linear matrix inequalities; Bounded input bounded output

\section{Introduction}

As one of the important nonlinear systems, the Lurie system can be deemed to consist of the linear forward path part, and the nonlinear feedback path part which satisfies the nonlinear bounded constraints. Since the pioneering work in the last century by Lurie [1, 2], much related research has been carried out [3-7]. For instance, [8] studied the indirect regulation on a nonlinear system with delay argument; [9] investigated the stabilization on a nonlinear system with time delay. These results have possible applications in fields such as complex networks and chaotic systems which are Lurie systems [10-16].

The analysis on bounded input bounded output (BIBO) stability of systems is very important for its possible application in single/double loop $\Sigma$ modulators, issues connected with bilinear input/output maps and so on, and they have received a lot of attention from scholars. For instance, [17] studied the BIBO stability of 2D discrete delayed systems, [18] researched the BIBO stability of fractional systems, [19] investigated study the BIBO stability of switched uncertain neutral systems, [20] concerned the BIBO stability of perturbed interconnected power systems, and [21] focused on the BIBO stability of feedback control systems. However, the results on BIBO stability for the Lurie system is seldom found at present. These motivate our research.

In addition, time-varying delay exists in practical systems widely [22-28], which will make impacts to the stability of Lurie systems. The requirement that the derivative of time-varying delay is less than 1 will restrict the applied scope of the criteria.

With the above concerns, the problem on the bounded input bounded output stability for the Lurie system with time-varying delay will be discussed. The remainder of this paper is organized as follows. Model description and preliminaries will be presented in Section 2. Based on Lyapunov function constructed and linear matrix inequalities [29], the bounded

(c) The Author(s) 2018. This article is distributed under the terms of the Creative Commons Attribution 4.0 International License (http://creativecommons.org/licenses/by/4.0/), which permits unrestricted use, distribution, and reproduction in any medium, provided you give appropriate credit to the original author(s) and the source, provide a link to the Creative Commons license, and indicate if changes were made. 
input bounded output criteria will be derived in Section 3. Typical numerical examples will be included to show the effectiveness of theoretical results obtained in Section 4. Finally, the paper will be concluded in Section 5.

Notation $R$ denotes the set of real numbers. $R_{+}$denotes the set of nonnegative real numbers. $R^{n}$ denotes the set of $n$-dimensional real column vectors. $R^{n \times n}$ denotes the set of $n \times n$ real matrix. $*$ denotes the symmetric part in matrix. $A>0$ means that $A$ is a real symmetric positive definitive matrix. $I$ denotes the identity matrix with appropriate dimensions. $\operatorname{diag}\{\cdots\}$ denotes the diagonal matrix. $\lambda_{\min }(\cdot)$ denotes the minimum eigenvalue of a matrix. $\lambda_{\max }(\cdot)$ denotes the maximum eigenvalue of a matrix. $L_{\infty}^{n}$ denotes the set of bounded function $r: R_{+} \rightarrow R_{n \times n}$ with norm $\|r\|_{\infty}=\sup _{t_{0} \leq t<\infty}\|r(t)\|<+\infty$. sup denotes the supremum. $\|\cdot\|_{\infty}$ denotes the infinite norm.

\section{Model description and preliminaries}

Consider the following Lurie system with time-varying delay:

$$
\left\{\begin{array}{l}
\dot{x}(t)=A x(t)+B x(t-\tau(t))+D f(t, z(t))+H u(t) \\
z(t)=L x(t)+N x(t-\tau(t)) \\
u(t)=G x(t)+r(t) \\
Y(t)=J x(t) \\
x\left(t_{0}+\theta\right)=\varphi(\theta), \quad \theta \in\left[\begin{array}{ll}
-h & 0
\end{array}\right]
\end{array}\right.
$$

where $x(t) \in R^{n}$ is the state vector of the system, $0 \leq \tau(t) \leq h$ is the time-varying delay, $\varphi(\theta) \in L_{n, h}$ is the initial condition of the system. $u(t) \in R^{l}$ is the control input, $Y(t) \in R^{m}$ is the system output, $r(t) \in R^{l}$ is the reference input, $f(t, z(t)) \in R^{n}$ is the system's nonlinear term, satisfying the bounded sector constraint,

$$
f^{T}(t, z(t))(f(t, z(t))-K z(t)) \leq 0
$$

where $K$ is a positive scalar.

Hence,

$$
-2 f^{T}(t) f(t)+2 f^{T}(t) K(L x(t)+N x(t-\tau(t))) \geq 0 .
$$

Lurie system (1) can be represented as

$$
\left\{\begin{array}{l}
\dot{x}(t)=y(t) \\
y(t)=A x(t)+B x(t-\tau(t))+D f(t, z(t))+H u(t), \\
z(t)=L x(t)+N x(t-\tau(t)), \\
u(t)=G x(t)+r(t), \\
Y(t)=J x(t), \\
x\left(t_{0}+\theta\right)=\varphi(\theta), \quad \theta \in\left[\begin{array}{ll}
-h & 0
\end{array}\right] .
\end{array}\right.
$$

In the paper, the following lemma and definitions are needed. 
Lemma 1 ([30]) For any constant matrices $E, G$ and $F$ with appropriate dimensions, $F^{T} F \leq k I, k$ is a positive scalar, then

$$
2 x^{T} E F G y \leq c x^{T} E E^{T} x+\frac{k}{c} y^{T} G^{T} G y,
$$

where $c$ is a positive scalar, $x \in R^{n}$ and $y \in R^{n}$.

Definition 1 ([31]) A real-valued vector $r(t) \in L_{\infty}^{n}$, if $\|r\|_{\infty}=\sup _{t_{0} \leq t<\infty}\|r(t)\|<+\infty$.

Definition 2 ([31]) The control system with reference input $r(t)$ is bounded input bounded output stable, if there exist some positive constants $\theta_{1}, \theta_{2}$, satisfies

$$
\|Y(t)\| \leq \theta_{1}\|r\|_{\infty}+\theta_{2}
$$

for every reference input $r(t) \in L_{\infty}^{n}$.

\section{Main results}

In this section, based on the Lyapunov method and linear matrix inequality techniques, the following stability criteria are derived.

Theorem 1 For the given positive scalars $h$ and $k$, Lurie system (1) is bounded input bounded output stable, if there exist matrices $P, R, Q, S, P_{2}, P_{3}, U, V, W$, and positive scalar $\sigma$, such that

$$
\begin{aligned}
& \Sigma+\Xi+\Xi^{T}+h e^{k h} W<0, \\
& {\left[\begin{array}{cc}
W & U, \\
* & S-R_{22}
\end{array}\right]>0,} \\
& {\left[\begin{array}{cc}
W & V \\
* & S
\end{array}\right]>0}
\end{aligned}
$$

where

$$
\begin{aligned}
& \Sigma=\left[\begin{array}{cccccc}
\Sigma_{1,1} & \Sigma_{1,2} & \Sigma_{1,3} & \Sigma_{1,4} & \Sigma_{1,5} & \Sigma_{1,6} \\
* & \Sigma_{2,2} & \Sigma_{2,3} & \Sigma_{2,4} & \Sigma_{2,5} & \Sigma_{2,6} \\
* & * & \Sigma_{3,3} & \Sigma_{3,4} & 0 & 0 \\
* & * & * & \Sigma_{4,4} & 0 & 0 \\
* & * & * & * & \Sigma_{5,5} & 0 \\
* & * & * & * & * & \Sigma_{6,6}
\end{array}\right], \\
& \Sigma_{1,1}=P_{2} A+A^{T} P_{2}^{T}+P_{2} H G+G^{T} H^{T} P_{2}^{T}+k P+Q, \quad \Sigma_{2,4}=P_{3} D, \\
& \Sigma_{1,2}=P-P_{2}+A^{T} P_{3}^{T}+G^{T} H^{T} P_{3}^{T}, \quad \Sigma_{3,4}=N^{T} K^{T}, \\
& \Sigma_{2,2}=h e^{k h} S-P_{3}-P_{3}^{T}, \quad \Sigma_{4,4}=-2 I, \\
& \Sigma_{1,3}=P_{2} B+R_{12}^{T}, \quad \Sigma_{5,5}=-Q e^{-k h}, \\
& \Sigma_{2,3}=P_{3} B, \quad \Sigma_{1,6}=P_{2} H,
\end{aligned}
$$




$$
\begin{aligned}
& \Sigma_{3,3}=h R_{11}-R_{12}-R_{12}^{T}, \quad \Sigma_{2,6}=P_{3} H, \\
& \Sigma_{1,4}=P_{2} D+L^{T} K^{T}, \quad \Sigma_{6,6}=-\sigma I, \\
& \Xi=\left[\begin{array}{llllll}
U & 0 & -U+V & 0 & -V & 0
\end{array}\right] .
\end{aligned}
$$

Proof Choose the Lyapunov-Krasovskii functional [32] as

$$
V(t)=V_{1}(t)+V_{2}(t)+V_{3}(t)+V_{4}(t)+V_{5}(t)
$$

where

$$
\begin{aligned}
& V_{1}(t)=\left(x^{T}(t) \quad y^{T}(t)\right)\left[\begin{array}{ll}
I & 0 \\
0 & 0
\end{array}\right]\left[\begin{array}{cc}
P & 0 \\
P_{2}^{T} & P_{3}^{T}
\end{array}\right]\left(\begin{array}{ll}
x^{T}(t) & \left.y^{T}(t)\right)^{T},
\end{array}\right. \\
& V_{2}(t)=\int_{-h}^{0} \int_{t+\beta}^{t} y^{T}(\alpha) e^{k(\alpha-t+h)} S y(\alpha) d \alpha d \beta \text {, } \\
& V_{3}(t)=\int_{-h}^{t} \int_{\beta-\tau(\beta)}^{\beta} \eta^{T} e^{k(\beta-t)} R \eta d \alpha d \beta, \\
& V_{4}(t)=\int_{-h}^{0} \int_{t+\beta}^{t} \xi^{T}(\alpha) e^{k(\alpha-t+h)} W \xi(\alpha) d \alpha d \beta, \\
& V_{5}(t)=\int_{t-h}^{t} x^{T}(s) e^{k(s-t)} Q x(s) d s, \\
& \eta=\left[\begin{array}{ll}
x(\beta-\tau(\beta)) & y(\alpha)
\end{array}\right]^{T}, \\
& \xi=\left[\begin{array}{llllll}
x^{T}(t) & y^{T}(t) & x^{T}(t-\tau(t)) & f^{T}(t) & x^{T}(t-h) & r^{T}(t)
\end{array}\right]^{T} .
\end{aligned}
$$

The derivative of $V(t)$ along trajectory of system (2) is given by

$$
\dot{V}(t)=\dot{V}_{1}(t)+\dot{V}_{2}(t)+\dot{V}_{3}(t)+\dot{V}_{4}(t)+\dot{V}_{5}(t)
$$

where

$$
\begin{aligned}
& \dot{V}_{1}(t)=2\left[\begin{array}{ll}
x^{T}(t) & y^{T}(t)
\end{array}\right]\left[\begin{array}{cc}
g(t) P_{i} & P_{2} \\
0 & P_{3}
\end{array}\right]\left[\begin{array}{c}
y(t) \\
0
\end{array}\right] \\
& =2 g(t) x^{T}(t) P_{i} y(t)+2\left(x^{T}(t) P_{2}+y^{T}(t) P_{3}\right)(-y(t)+(A+H G) x(t) \\
& +B x(t-\tau(t))+D f(t)+H r(t)), \\
& \dot{V}_{2}(t)=h y^{T}(t) e^{k h} S y(t)-\int_{t-\tau(t)}^{t} y^{T}(s) e^{k(s-t+h)} S y(s) d s-\int_{t-h}^{t-\tau(t)} y^{T}(s) e^{k(s-t+h)} S y(s) d s \\
& -k V_{2}(t) \\
& \leq h y^{T}(t) e^{k h} S y(t)-\int_{t-\tau(t)}^{t} y^{T}(s) S y(s) d s-\int_{t-h}^{t-\tau(t)} y^{T}(s) S y(s) d s-k V_{2}(t), \\
& \dot{V}_{3}(t)=\tau(t) x^{T}(t-\tau(t)) R_{11} x(t-\tau(t))+2 x^{T}(t-\tau(t)) R_{12} x(t) \\
& -2 x^{T}(t-\tau(t)) R_{12} x(t-\tau(t))+\int_{t-\tau(t)}^{t} y(s) R_{22} y(s) d s-k V_{3}(t)
\end{aligned}
$$


Wang et al. Advances in Difference Equations （2018） 2018:57

Page 5 of 13

$$
\begin{aligned}
\leq & h x^{T}(t-\tau(t)) R_{11} x(t-\tau(t))+2 x^{T}(t) R_{12}^{T} x(t-\tau(t)) \\
& -2 x^{T}(t-\tau(t)) R_{12} x(t-\tau(t))+\int_{t-\tau(t)}^{t} y(s) R_{22} y(s) d s-k V_{3}(t), \\
\dot{V}_{4}(t)= & h \xi^{T}(t) e^{k h} W \xi(t)-\int_{t-\tau(t)}^{t} \xi^{T}(s) e^{k(s-t+h)} W \xi(s) d s \\
& -\int_{t-h}^{t-\tau(t)} \xi^{T}(s) e^{k(s-t+h)} W \xi(s) d s-k V_{4}(t) \\
\leq & h \xi^{T}(t) e^{k h} W \xi(t)-\int_{t-\tau(t)}^{t} \xi^{T}(s) W \xi(s) d s-\int_{t-h}^{t-\tau(t)} \xi^{T}(s) W \xi(s) d s-k V_{4}(t), \\
\dot{V}_{5}(t)= & x^{T}(t) Q x(t)-x^{T}(t-h) e^{-k h} Q x(t-h)-k V_{5}(t) .
\end{aligned}
$$

According to the Leibniz-Newton formula [33]

$$
\begin{aligned}
& 2 \xi^{T} U\left[x(t)-x(t-\tau(t))-\int_{t-\tau(t)}^{t} y^{T}(s) d s\right]=0, \\
& 2 \xi^{T} V\left[x(t-\tau(t))-x(t-h)-\int_{t-h}^{t-\tau(t)} y^{T}(s) d s\right]=0 .
\end{aligned}
$$

Therefore,

$$
\begin{aligned}
\dot{V}(t) \leq & \xi^{T}\left(\Sigma+\Omega+\Omega^{T}+h e^{k h} W\right) \xi-\int_{t-\tau(t)}^{t} \zeta^{T} \Phi_{1} \zeta d s-\int_{t-h}^{t-\tau(t)} \zeta^{T} \Phi_{2} \zeta d s \\
& -k V(t)+\sigma\|r(t)\|_{\infty}^{2}
\end{aligned}
$$

where

$$
\begin{aligned}
& \zeta=\left[\begin{array}{ll}
\xi^{T} & y^{T}(s)
\end{array}\right]^{T}, \\
& \Phi_{1}=\left[\begin{array}{cc}
W & U \\
* & S-R_{22}
\end{array}\right], \\
& \Phi_{2}=\left[\begin{array}{ll}
W & V \\
* & S
\end{array}\right] .
\end{aligned}
$$

According to (5)

$$
\dot{V}(t) \leq-k V(t)+\sigma\|r(t)\|_{\infty}^{2}
$$

We will have the following formula:

$$
\left(V(t) e^{k t}\right)^{\prime} \leq(\dot{V}(t)+k V(t)) e^{k t} \leq \sigma\|r(t)\|_{\infty}^{2} e^{k t} .
$$

We integrate the above inequality from $t_{0}$ to $t$

$$
V(t) e^{k t} \leq V\left(t_{0}\right) e^{k t_{0}}+\sigma\|r(t)\|_{\infty}^{2} \int_{t_{0}}^{t} e^{k s} d s
$$


We will obtain

$$
\lambda_{\min }(P)\|x\|^{2} \leq V(t) \leq V\left(t_{0}\right) e^{-k\left(t-t_{0}\right)}+\sigma\|r(t)\|_{\infty}^{2} \int_{t_{0}}^{t} e^{-k(t-s)} d s .
$$

Consider

$$
\begin{aligned}
\int_{t_{0}}^{t} e^{-k(t-s)} d s & =e^{-k t} \int_{t_{0}}^{t} e^{k s} d s \\
& =e^{-k t} \frac{\left(e^{k t}-e^{k t_{0}}\right)}{k} \\
& =\frac{1}{k}-\frac{e^{k\left(t_{0}-t\right)}}{k} \\
& \leq \frac{1}{k} .
\end{aligned}
$$

One can get

$$
\lambda_{\min }(P)\|x\|^{2} \leq V\left(t_{0}\right) e^{-k\left(t-t_{0}\right)}+\frac{\sigma\|r(t)\|_{\infty}^{2}}{k} .
$$

Let us define

$$
\psi=\max \left\{\sup _{h \leq \theta \leq 0}\left\|\varphi\left(t_{0}+\theta\right)\right\|, \sup _{h \leq \theta \leq 0}\left\|\varphi^{\prime}\left(t_{0}+\theta\right)\right\|\right\} .
$$

According to the definition of $V(t)$, we have

$$
V\left(t_{0}\right) \leq\left[\lambda_{\max }(P)+h^{2} e^{k h} \lambda_{\max }(S)+h^{2} \lambda_{\max }(R)+h^{2} e^{k h} \lambda_{\max }(W)+h \lambda_{\max }(Q)\right] \psi^{2} .
$$

The following inequality can be concluded:

$$
\begin{aligned}
\|x\|^{2} & \leq \frac{a}{\lambda_{\min }(P)} \psi^{2}+\frac{\sigma}{k \lambda_{\min }(P)}\|r(t)\|_{\infty}^{2} \\
& \leq\left(\sqrt{\frac{a}{\lambda_{\min }(P)}} \psi+\sqrt{\frac{\sigma}{k \lambda_{\min }(P)}}\|r(t)\|_{\infty}\right)^{2},
\end{aligned}
$$

where

$$
a=\lambda_{\max }(P)+h^{2} e^{k h} \lambda_{\max }(S)+h^{2} \lambda_{\max }(R)+h^{2} e^{k h} \lambda_{\max }(W)+h \lambda_{\max }(Q)>0 .
$$

We will obtain

$$
\|Y\| \leq\|J\|\|x\| \leq \theta_{1}+\theta_{2}\|r(t)\|_{\infty}
$$

where

$$
\begin{aligned}
& \theta_{1}=\|J\| \sqrt{\frac{a}{\lambda_{\min }(P)}} \psi, \\
& \theta_{2}=\|J\| \sqrt{\frac{\sigma}{k \lambda_{\min }(P)}} .
\end{aligned}
$$


Hence, Lurie system (1) is bounded input bounded output stable. The proof of Theorem 1 is thus completed.

\section{Extension}

Next, we consider Lurie system (1) with nonlinear term, which satisfies the following bounded sector constraint:

$$
\left(f^{T}(t, z(t))-K_{1} z(t)\right)\left(f(t, z(t))-K_{2} z(t)\right) \leq 0
$$

where $K_{1}$ and $K_{2}$ are positive scalars, such that

$$
K_{2}>K_{1}
$$

Let

$$
F^{T}(t, z(t))=f^{T}(t, z(t))-K_{1} z(t) .
$$

We have

$$
F^{T}(t, z(t))(F(t, z(t))-K z(t)) \leq 0
$$

where

$$
K=K_{2}-K_{1} \text {. }
$$

The following inequality can be derived:

$$
-2 F^{T}(t) F(t)+2 F^{T}(t) K(L x(t)+N x(t-\tau(t))) \geq 0 .
$$

Therefore Lurie system (1) can be transformed as

$$
\left\{\begin{array}{l}
\dot{x}(t)=\bar{A} x(t)+\bar{B} x(t-\tau(t))+D F(t, z(t))+H u(t) \\
z(t)=L x(t)+N x(t-\tau(t)) \\
u(t)=G x(t)+r(t) \\
Y(t)=J x(t) \\
x\left(t_{0}+\theta\right)=\varphi(\theta), \quad \theta \in\left[\begin{array}{ll}
-h & 0
\end{array}\right]
\end{array}\right.
$$

where

$$
\begin{aligned}
& \bar{A}=A+D K_{1} L, \\
& \bar{B}=B+D K_{1} N .
\end{aligned}
$$

Then based on Theorem 1, the following theoretical result can be concluded. 
Theorem 2 For the given positive scalars $h$ and $k$, Lurie system (6) is bounded input bounded output stable, if there exist matrices $P, R, Q, S, P_{2}, P_{3}, U, V, W$, and positive scalar $\sigma$, such that

$$
\begin{aligned}
& \Sigma+\Xi+\Xi^{T}+h e^{k h} W<0, \\
& {\left[\begin{array}{cc}
W & U \\
* & S-R_{22}
\end{array}\right]>0,} \\
& {\left[\begin{array}{cc}
W & V \\
* & S
\end{array}\right]>0}
\end{aligned}
$$

where

$$
\begin{aligned}
& \Sigma=\left[\begin{array}{cccccc}
\Sigma_{1,1} & \Sigma_{1,2} & \Sigma_{1,3} & \Sigma_{1,4} & \Sigma_{1,5} & \Sigma_{1,6} \\
* & \Sigma_{2,2} & \Sigma_{2,3} & \Sigma_{2,4} & \Sigma_{2,5} & \Sigma_{2,6} \\
* & * & \Sigma_{3,3} & \Sigma_{3,4} & 0 & 0 \\
* & * & * & \Sigma_{4,4} & 0 & 0 \\
* & * & * & * & \Sigma_{5,5} & 0 \\
* & * & * & * & * & \Sigma_{6,6}
\end{array}\right] \\
& \Sigma_{1,1}=P_{2}\left(A+D K_{1} L\right)+\left(A+D K_{1} L\right)^{T} P_{2}^{T}+P_{2} H G+G^{T} H^{T} P_{2}^{T}+k P+Q, \quad \Sigma_{2,4}=P_{3} D, \\
& \Sigma_{1,2}=P-P_{2}+\left(A+D K_{1} L\right)^{T} P_{3}^{T}+G^{T} H^{T} P_{3}^{T}, \quad \Sigma_{3,4}=N^{T} K^{T}, \\
& \Sigma_{2,2}=h e^{k h} S-P_{3}-P_{3}^{T}, \quad \Sigma_{4,4}=-2 I \text {, } \\
& \Sigma_{1,3}=P_{2}\left(B+D K_{1} N\right)+R_{12}^{T}, \quad \Sigma_{5,5}=-Q e^{-k h}, \\
& \Sigma_{2,3}=P_{3}\left(B+D K_{1} N\right), \quad \Sigma_{1,6}=P_{2} H, \\
& \Sigma_{3,3}=h R_{11}-R_{12}-R_{12}^{T}, \quad \Sigma_{2,6}=P_{3} H, \\
& \Sigma_{1,4}=P_{2} D+L^{T} K^{T}, \quad \Sigma_{6,6}=-\sigma I, \\
& \Xi=\left[\begin{array}{llllll}
U & 0 & -U+V & 0 & -V & 0
\end{array}\right] \text {. }
\end{aligned}
$$

\section{Simulation}

In this section, some typical simulation examples will be included to verify the correctness of the theoretical results.

Consider the following Lurie system:

$$
\left\{\begin{array}{l}
\dot{x}(t)=A x(t)+B x(t-\tau(t))+D f(t, z(t))+H u(t), \\
z(t)=L x(t)+N x(t-\tau(t)), \\
u(t)=G x(t)+r(t), \\
Y(t)=J x(t), \\
x\left(t_{0}+\theta\right)=\varphi(\theta), \quad \theta \in\left[\begin{array}{ll}
-h & 0
\end{array}\right]
\end{array}\right.
$$

with

$$
A=\left[\begin{array}{cc}
-1 & 0.5 \\
0.5 & -2
\end{array}\right], \quad B=\left[\begin{array}{cc}
-0.4 & 0 \\
0.3 & -0.4
\end{array}\right], \quad D=\left[\begin{array}{cc}
-0.4 & 0.3 \\
0 & -0.3
\end{array}\right],
$$




$$
\begin{aligned}
& H=\left[\begin{array}{cc}
0.4 & 0 \\
0 & 0.4
\end{array}\right], \quad L=\left[\begin{array}{cc}
0.2 & 0 \\
0 & 0.2
\end{array}\right], \quad N=\left[\begin{array}{cc}
0.3 & 0 \\
0 & 0.3
\end{array}\right], \\
& G=\left[\begin{array}{cc}
0.2 & 0 \\
0 & 0.2
\end{array}\right], \quad J=\left[\begin{array}{ll}
1 & 0 \\
0 & 1
\end{array}\right], \\
& r(t)=\left[2 \cos (2 t) \sin \left(\frac{e^{t}}{t+1}\right) ; \sin (2 t) \cos \left(e^{t}\right)\right], \\
& f(t, z)=[|z+1|+|z-1|] / 2, \\
& \tau(t)=1+0.5 \sin ^{2}(6 t), \quad K=I .
\end{aligned}
$$

Let $h=1.5, k=0.1$, according to Theorem 1 , we can get

$$
\begin{array}{ll}
P=\left[\begin{array}{ll}
6.9034 & 0.3387 \\
0.3387 & 6.4575
\end{array}\right], & P_{2}=\left[\begin{array}{ll}
4.6360 & 0.9507 \\
1.0990 & 2.8319
\end{array}\right], \\
P_{3}=\left[\begin{array}{ll}
3.6444 & 0.6788 \\
0.9099 & 2.3317
\end{array}\right], & R_{11}=\left[\begin{array}{ll}
0.4741 & 0.0962 \\
0.0962 & 0.3748
\end{array}\right], \\
R_{12}=\left[\begin{array}{ll}
0.5787 & 0.1252 \\
0.1452 & 0.4494
\end{array}\right], & R_{22}=\left[\begin{array}{ll}
1.0521 & 0.3290 \\
0.3290 & 0.7846
\end{array}\right], \\
S=\left[\begin{array}{ll}
2.0780 & 0.4701 \\
0.4701 & 1.3947
\end{array}\right], & Q=\left[\begin{array}{cc}
3.4753 & -0.6448 \\
-0.6448 & 4.3384
\end{array}\right], \\
\sigma & =6.0819 .
\end{array}
$$

Remark 1 For the given example, $1 \leq \dot{\tau}(t) \leq 1.5$, our criterion is still available because it is independent of the derivative of the time-varying delay of the system.

Remark 2 When the parameter $k$ is fixed, the allowable upper bound $h_{\max }$ of time delay $h$ of Lurie system (1) can be determined by solving the following optimization problem based on LMI method:

$$
\left\{\begin{array}{l}
h_{\max }=\max _{\mathrm{k} \in[0,0.3]}\{h\} \\
\text { when LMI (5) is satisfied. }
\end{array}\right.
$$

For the given example, we can get Table 1.

Remark 3 Table 1 shows the relationship between the parameter $k$ and the allowable upper bound $h_{\max }$ of time delay. It can be seen that the allowable upper bound $h_{\max }$ of time delay decreases with the increase of the parameter $k$, and it takes the maximum value 2.5973 when $k$ is zero.

Next, set the system initial state $\varphi(\theta)=[-1.5 ; 1.5]^{T}, t \in(-1.5,0)$ and the numerical simulation step $0.001 \mathrm{~s}$. Corresponding numerical simulation results are shown in Figures 1-4.

Table 1 Relationship between $k$ and $h_{\max }$

\begin{tabular}{llll}
\hline$k=0$ & $k=0.1$ & $k=0.2$ & $k=0.3$ \\
$h_{\max }=2.5973$ & $h_{\max }=2.1693$ & $h_{\max }=1.8842$ & $h_{\max }=1.6736$ \\
\hline
\end{tabular}




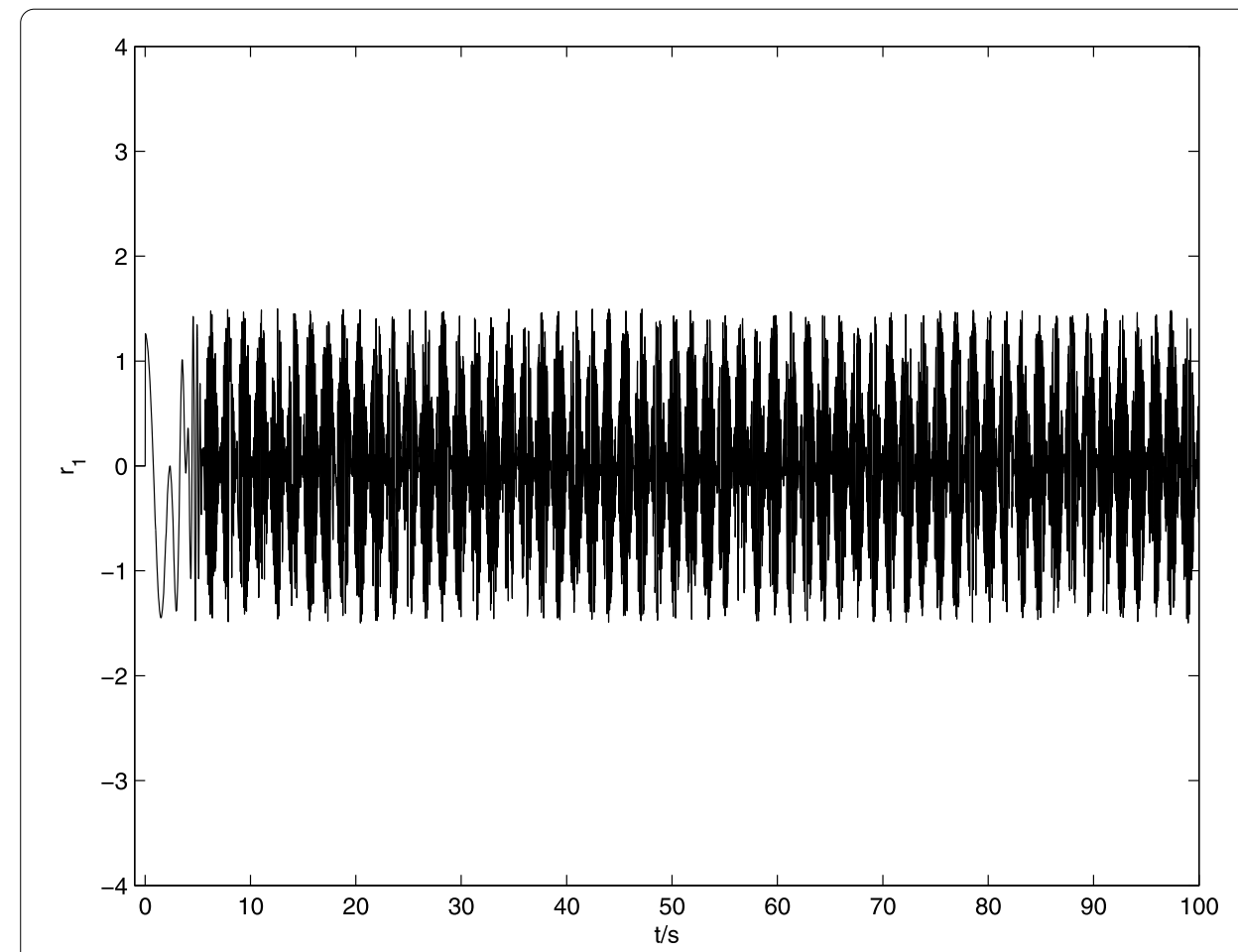

Figure 1 Time response of reference input variable $r_{1}(t)$ of the Lurie system

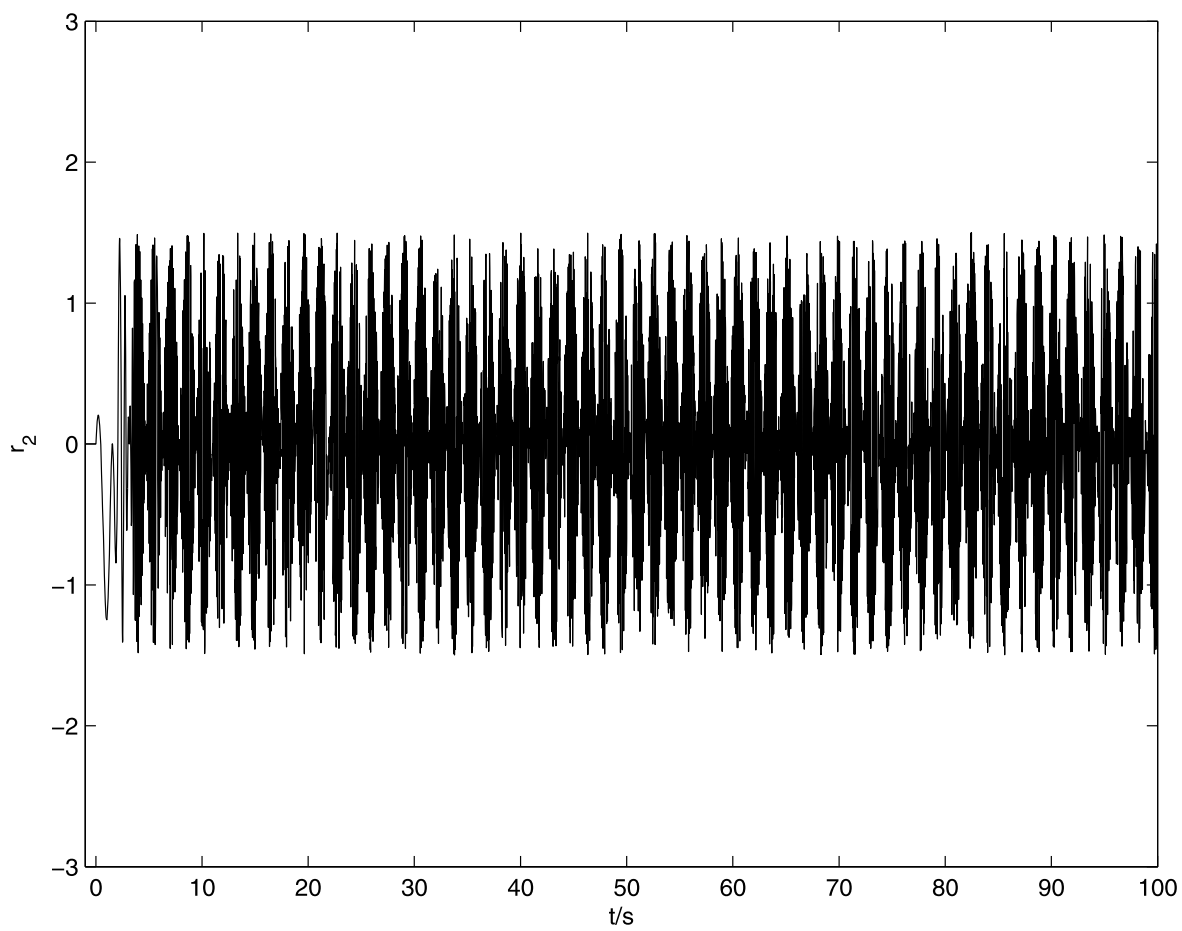

Figure 2 Time response of reference input variable $r_{2}(t)$ of the Lurie system 
Wang et al. Advances in Difference Equations （2018） 2018:57

Page 11 of 13

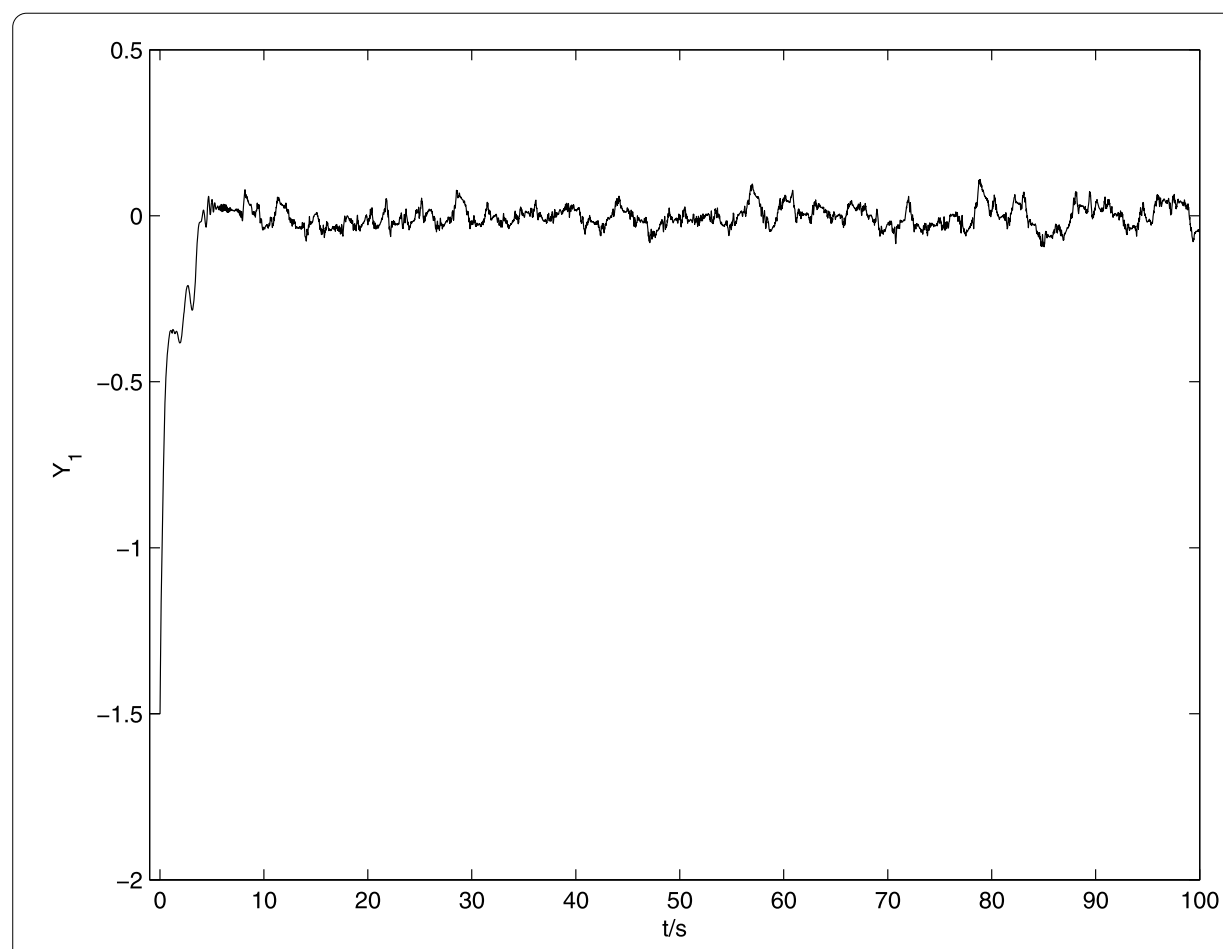

Figure 3 Time response of output variable $Y_{1}(t)$ of the Lorie system

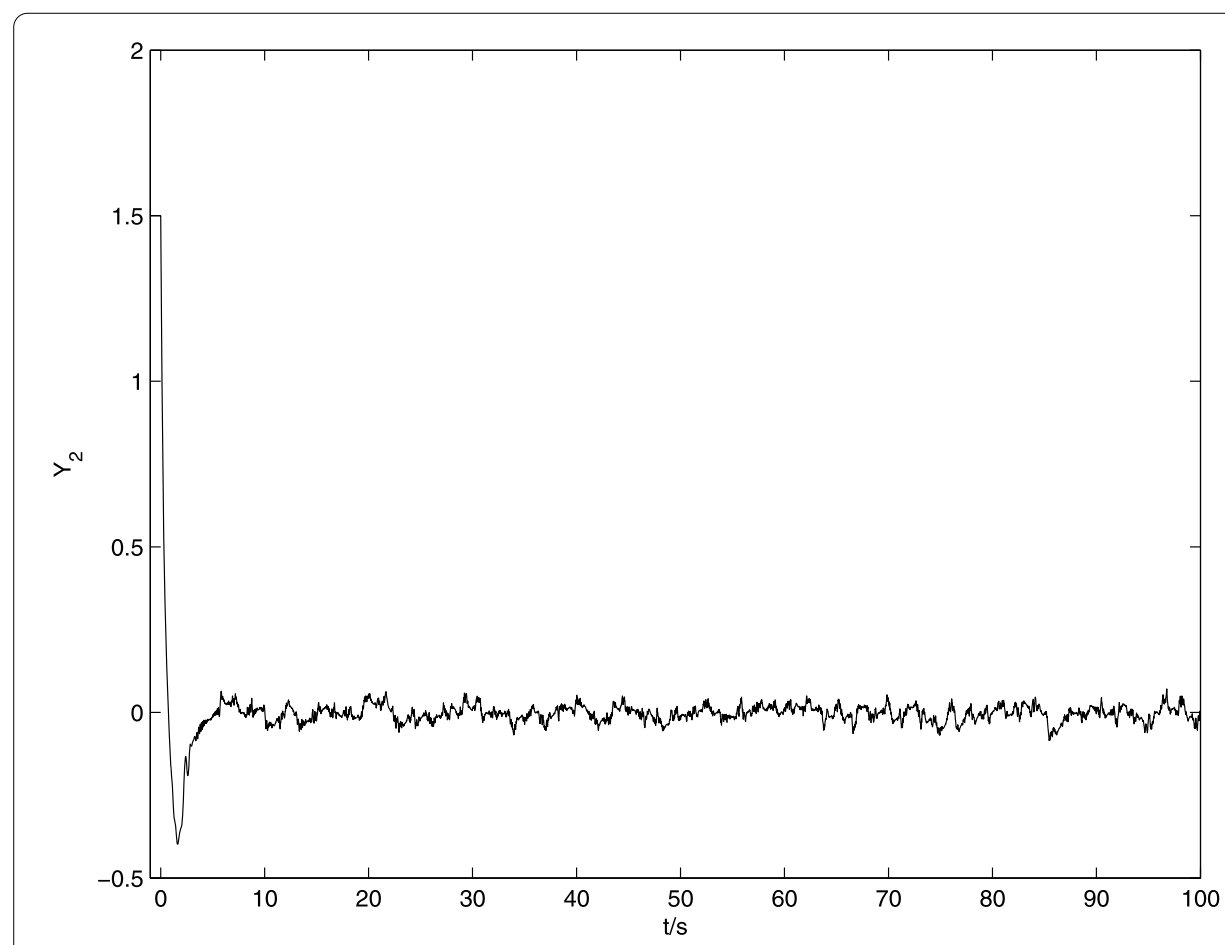

Figure 4 Time response of output variable $Y_{2}(t)$ of the Lorie system 
Remark 4 Figures 1 and 2 depict the time response of reference input variables $r_{1}(t)$ and $r_{2}(t)$ of the Lurie system. It can be seen that the motion of the system reference input variables are restricted in a set range. Figures 3 and 4 depict the time response of output variables $Y_{1}(t)$ and $Y_{2}(t)$ of the Lurie system. It can be seen that the system output variables move within a bounded range. The Lurie system in the example is bounded input bounded output stable.

\title{
6 Conclusions
}

In this paper, we have studied the bounded input bounded output stability for the Lurie system with time-varying delay. Based on the Lyapunov method and linear matrix inequality technology, new bounded input bounded output stability criteria for the Lurie system have been derived. A typical numerical simulation example has been included to verify the correctness of the presented theoretical results.

\author{
Acknowledgements \\ This work was partially supported by the Project of Education Department of Sichuan Province (15ZA0142), the Chunhui \\ Plan Project of Ministry of Education (Z2015114), the Graduate Innovation Fund (ycjj2017171), the National Natural \\ Science Foundation of China (61703150, 11626093), the Natural Science Foundation of Hubei Provinces of China \\ (2016CFB211). \\ Competing interests \\ The authors declare that they have no competing interests. \\ Authors' contributions \\ All authors read and approved the final manuscript.

\section{Author details} \\ ${ }^{1}$ Key Laboratory of Fluid and Power Machinery of the Ministry of Education, School of Electrical Engineering and \\ Electronic Information, Xihua University, Chengdu, P.R. China. ${ }^{2}$ School of Applied Mathematics, University Electronic \\ Science and Technology of China, Chengdu, P.R. China. ${ }^{3}$ School of Science, Hubei University for Nationalities, Enshi, \\ P.R. China.
}

\section{Publisher's Note}

Springer Nature remains neutral with regard to jurisdictional claims in published maps and institutional affiliations.

Received: 17 August 2017 Accepted: 25 January 2018 Published online: 14 February 2018

\section{References}

1. Lur'e, A., Postnikov, V.: On the theory of stability of control system. Prikl. Mat. Meh. 8, 283-286 (1944) (in Russian)

2. Lur'e, A.: Some Nonlinear Problem in the Theory of Automatic Control. H. M. Stationery Office, London (1957)

3. Shatyrko, A., Khusainov, D.: On the interval stability of weak-nonlinear control systems with aftereffect. Sci. World J. 2016, Article ID 6490826 (2016)

4. Aizerman, M., Gantmacher, F.: Absolute Stability of Regulator Systems. Holden-Day, San Francisco (1964)

5. Shatyrko, A., Nooijen, R., Kolechkina, A., Khusainov, D.: Stabilization of neutral-type indirect control systems to absolute stability state. Adv. Differ. Equ. 2015, 64 (2015)

6. Shatyrko, A.: Interval stability of nonlinear control systems with aftereffect. In: Post-Conference Proceedings of Selected Papers (extended version MITAV-2015), Brno, Czech Republic, pp. 122-132 (2015)

7. Khusainov, D., Shatyrko, A.: Absolute stability of multi-delay regulation systems. J. Autom. Inf. Sci. 27, 3-4 (1995)

8. Shatyrko, A., Khusainov, D.: Absolute stability conditions construction of non-direct regulator systems by Lyapunov-Krasovskiy functional. Bull. Kyiv Univ., Ser. Phys. Math. 4, 145-152 (2009) (In Ukrainian)

9. Shatyrko, A., Diblik, J., Khusainov, D., Ruzickova, M.: Stabilization of Lur'e-type nonlinear control systems by Lyapunov-Krasovski functionals. Adv. Differ. Equ. 2012, 229 (2012)

10. Wang, B., Shi, P., Karimi, H.: Robust $H_{\infty}$ synchronization of a hyper-chaotic system with disturbance input. Nonlinear Anal., Real World Appl. 14, 1487-1495 (2013)

11. Shen, H., Park, J., Wu, Z.: Finite-time synchronization control for uncertain Markov jump neural networks with input constraints. Nonlinear Dyn. 77, 1709-1720 (2014)

12. Wang, B., Zou, F., Cheng, J.: A memristor-based chaotic system and its application in image encryption. Optik, Int. J. Light Electron Opt. 154, 538-544 (2018)

13. Wang, J., Su, L., Shen, H., Wu, Z., Park, J.: Mixed $H_{\infty}$ /passive sampled-data synchronization control of complex dynamical networks with distributed coupling delay. J. Franklin Inst. 354, 1302-1320 (2017)

14. Wang, B., Zhong, S.: Observer-based control on a chaotic system with unknowns and uncertainties. Optik, Int. J. Light Electron Opt. 137, 167-174 (2017) 
15. Shen, H., Zhu, Y., Zhang, L., Park, J.: Extended dissipative state estimation for Markov jump neural networks with unreliable links. IEEE Trans. Neural Netw. Learn. Syst. 28, 346-358 (2017)

16. Wang, B., Zhong, S., Dong, X.: On the novel chaotic secure communication scheme design. Commun. Nonlinear Sci. Numer. Simul. 39, 108-117 (2016)

17. Wang, L., Chen, X., Gao, F.: Delay-range-dependent robust BIBO stabilization of $2 \mathrm{D}$ discrete delayed systems via LMI approach. IFAC Proc. Vol. 47, 10994-10999 (2014)

18. Abusaksaka, A., Partington, J.: BIBO stability of some classes of delay systems and fractional systems. Syst. Control Lett. 64, 43-46 (2014)

19. Xiong, L., Zhong, S., Ye, M.: Delay-dependent BIBO stability analysis of switched uncertain neutral systems. Math. Comput. Model. 53, 1607-1620 (2011)

20. Pham, T., Nahavandi, S., Trinh, H., Hien, L.: Decentralized bounded input bounded output stabilization of perturbed interconnected time-delay power systems with energy storages. Int. J. Electr. Power Energy Syst. 93, 51-64 (2017)

21. Awwad, E., Győri, I., Hartung, F.: BIBO stabilization of feedback control systems with time dependent delays. Appl. Math. Comput. 219, 3664-3676 (2012)

22. Malek-Zavarei, M., Jamshidi, M.: Time Delay Systems: Analysis, Optimization and Applications. North-Holland, Amsterdam (1987)

23. Wang, B., Yan, J., Cheng, J., Zhong, S.: New criteria of stability analysis for generalized neural networks subject to time-varying delayed signals. Appl. Math. Comput. 314, 322-333 (2017)

24. Rasvan, V.: Systems with monotone and slope restricted nonlinearities. Tatra Mt. Math. Publ. 48, 165-187 (2011)

25. Wang, B., Cheng, J., Al-Barakati, A., Fardoun, H.: A mismatched membership function approach to sampled-data stabilization for T-S fuzzy systems with time-varying delayed signals. Signal Process. 140, 161-170 (2017)

26. Cheng, J., Chang, X., Park, J., Li, H., Wang, H.: Fuzzy-model-based $H_{\infty}$ control for discrete-time switched systems with quantized feedback and unreliable links. Inf. Sci. 436, 181-196 (2018)

27. Rasvan, V: Stabilization and decoupling control applied in vehicle dynamics. IFAC Proc. Vol. 29, 97-102 (1996)

28. Wang, B., Cheng, J., Zhan, J.: A sojourn probability approach to fuzzy-model-based reliable control for switched systems with mode-dependent time-varying delays. Nonlinear Anal. Hybrid Syst. 26, 239-253 (2017)

29. Boyd, S., Ghaoui, L., Feron, E., Balakrishnan, V.: Linear Matrix Inequalities in Systems and Control Theory. SIAM, Philadelphia (1994)

30. Gu, K:: An integral inequality in the stability problem of time-delay systems. In: Proceedings of 39th IEEE CDC, Sydney, Australia, pp. 2805-2810 (2000)

31. Wu, H., Mizukami, K.: Robust stabilization of uncertain linear dynamical systems. Int. J. Syst. Sci. 24, 265-276 (1993)

32. El'sgol'ts, L., Norkin, S.: Introduction to the Theory of the Differential Equations with Deviating Argument. Academic Press, New York (1973)

33. Rajchakit, M., Niamsup, P., Rajchakit, G.: A switching rule for exponential stability of switched recurrent neural networks with interval time-varying delay. Adv. Differ. Equ. 2013, 44 (2013)

\section{Submit your manuscript to a SpringerOpen ${ }^{\circ}$ journal and benefit from:}

- Convenient online submission

- Rigorous peer review

- Open access: articles freely available online

- High visibility within the field

- Retaining the copyright to your article

Submit your next manuscript at $\gg$ springeropen.com 